\title{
Progressing in cable-in-conduit for fusion magnets: from ITER to low cost, high performance DEMO
}

\author{
D. Uglietti, K. Sedlak, R. Wesche, P. Bruzzone \\ EPFL-Swiss Plasma Center, 5232 - Villigen PSI, Switzerland (davide.uglietti@psi.ch) \\ L. Muzzi, A. della Corte \\ ENEA, 00044 Frascati, Italy
}

\begin{abstract}
The performance of ITER Toroidal Field (TF) conductors still have a significant margin for improvement because the effective strain between $-0.62 \%$ and $-0.95 \%$ limits the strands critical current between $15 \%$ and $45 \%$ of the maximum achievable. Prototype $\mathrm{Nb}_{3}$ Sn cable-in-conduit conductors have been designed, manufactured and tested in the frame of the EUROfusion DEMO activities. In these conductors the effective strain has shown a clear improvement with respect to the ITER conductors, reaching values between $-0.55 \%$ and $-0.28 \%$, resulting in a strand critical current which is two to three times higher than in ITER conductors. In terms of the amount of $\mathrm{Nb}_{3} \mathrm{Sn}$ strand required for the construction of the DEMO TF magnet system, such improvement may lead to a reduction of at least a factor two with respect to a similar magnet built with ITER type conductors; further saving of $\mathrm{Nb}_{3} \mathrm{Sn}$ is possible if graded conductors/windings are employed. In the best case the TF magnet of DEMO could require less $\mathrm{Nb}_{3} \mathrm{Sn}$ strands than the one of ITER, despite the larger size of DEMO. Moreover high performance conductors could be operated at higher fields than ITER TF conductors, enabling the construction of low cost, compact, high field tokamaks.
\end{abstract}

\section{Introduction}

Since the seventies it has been clear that future tokamak fusion power plants would require a superconducting magnet system because the electric power needed for a resistive one would be prohibitively high. Several superconducting tokamaks were built in the last four decades, most of them using NbTi because it is ductile, relatively cheap and the critical field is compatible with the requirement of low field tokamaks $(<4 \mathrm{~T}$ on the plasma). Large magnets cannot be built with single strands, the voltage during discharge being too large because of the large inductance; forced flow conductors have been extensively employed to build large fusion magnets, where various heat generation mechanism are present, because of the excellent heat removal capability; among forced flow conductors, cable-in-conduit (CIC) conductors have often been selected because of the large thermal stability.

$\mathrm{Nb}_{3} \mathrm{Sn}$ has also been employed for manufacturing few test coils (see [1] for a detailed overview), both by react and wind (R\&W) and by wind and react (W\&R). Two tokamaks have been built with $\mathrm{R} \& \mathrm{~W} \mathrm{Nb} \mathrm{N}_{3} \mathrm{Sn}$ : in $1986 \mathrm{a}$ monolithic conductor composed of a very large single strand $(10 \mathrm{~mm} \times 3.3 \mathrm{~mm})$ was used in the TF coils of TRIAM-1M (it still holds the record field in the plasma, 8 T) and in 1988 the TF coils of T-15 were built with flat cables composed of 11 strands. ITER opted for building a large Wind and React (W\&R) $\mathrm{Nb}_{3} \mathrm{Sn}$ tokamak for the first time; in fact the construction of KSTAR, which employed similar conductors, took place after ITER had already set the conductor design.

The tokamaks built up to now, including ITER, are mainly scientific instruments. The next step after ITER will be the transition from scientific instrument to prototype power plant, the so-called DEMO. EU-DEMO, according to the European roadmap to Fusion Electricity [2], will be the nearest-term reactor design capable of producing and injecting electricity in the grid. Quoting the roadmap: "... fusion will have to demonstrate the potential for competitive cost of electricity." and "... the perspective of economic electricity production from fusion has to be set as a target, e.g. minimizing the DEMO capital costs".

In order to minimize the DEMO capital cost it is not sufficient to reduce the cost of just one component, it is necessary to lower the cost of most of the expensive ones (civil engineering, $\mathrm{Nb}_{3} \mathrm{Sn}$ conductors, vacuum 
vessel, power converters, heating and current drive, magnet structures: see the cost estimate for ITER in [3]). Regarding the magnet system, which usually accounts for about $30 \%$ of the whole machine cost, not only the winding pack should be optimized, for example considering grading (using less superconductor where the magnetic field is lower), but also the $\mathrm{Nb}_{3} \mathrm{Sn}$ strands in the conductors should be used at the highest possible critical current because strands are a costly component: for example in ITER the $\mathrm{Nb}_{3} \mathrm{Sn}$ strands were expected to account for about $20 \%$ of the cost of the whole tokamak and about $10 \%$ of the total machine cost [3]. DEMO is more ambitious than ITER because the conductors should not only provide adequate performance during the entire life (objective that will be achieved in ITER), but they should also be cost-effective.

This paper summarizes the data collected during the testing of ITER conductors and compares this with the recent results obtained on the DEMO R\&D conductors in terms of effective strain fitting parameter and corresponding average critical current for the strands, assessing how far the performance (in terms of critical current, amount of strand and maximum operating field) of the ITER conductors are from the maximum values that $\mathrm{Nb}_{3} \mathrm{Sn}$ can deliver.

\section{Review and analysis of ITER conductor performance}

The focus in this paper is on the strain state of the $\mathrm{Nb}_{3} \mathrm{Sn}$ because this is the most important contribution coming from the conductor design. In fact the critical field, critical temperature, current density and strain dependence of the strands are set by the manufacturers, while the strain state (compressive and/or tensile) of the $\mathrm{Nb}_{3} \mathrm{Sn}$ filaments in the conductor, and thus the critical current, will depend on the conductor design. When $\mathrm{CIC}$ are manufactured with a jacket material whose thermal contraction is higher than the one of $\mathrm{Nb}_{3} \mathrm{Sn}$, the difference in thermal contraction generates a thermal compressive strain on the strands, whose magnitude and effects on $T_{c s}$ and $I_{c}$ are not easily estimated because of the complex cable structure.

\subsection{Strain in ITER CIC}

It is useful to summarize briefly how the understanding of the strain state in $\mathrm{Nb}_{3} \mathrm{Sn}$ CIC evolved since the beginning of the ITER project. The design of ITER TF conductor was set in the first half of the '90, when it was conjectured that a W\&R conductor would be more suitable [4] and the thermal strain was expected to be around $-0.75 \%$. In 2000, after Incoloy as jacket material was dropped in favour of 316LN, a thermal strain of $0.6 \%$ was assumed in full-size ITER TF prototype conductors [5] and the low $\mathrm{T}_{\mathrm{cs}}$ of these conductors was supposed to be caused by poor current distribution and design value was still maintained at $-0.6 \%$. The test of model coils in early 2000' showed that the conductor design could not guarantee the required performance [6] and it was proposed to reduce the void fraction and increase of current density in the strands. Since 2003 an "extra strain", which takes into account filament breakage [7] and additional compressive strain [8], has been added to the thermal strain.

Till mid 2000s it was assumed that the strain of the $\mathrm{Nb}_{3} \mathrm{Sn}$ filaments was uniform, but gradually the idea of non-homogeneous strain gained more and more relevance. In 2010 simulations of a model sub-cable [9] suggested the presence of a broad strain distribution instead of a uniform value. In 2012 and later [10] AC susceptibility measurements of the critical temperature of the $\mathrm{Nb}_{3} \mathrm{Sn}$ allowed to infer the strain distribution in few TF conductor samples, validating the result of the simulations: the strain distribution for TF conductors extends from about $-1 \%$ to the tensile region, and in average the value of the distribution peak was found to be $-0.37 \% \pm 0.05 \%$. In 2013-14 the relation between conductor design, $\mathrm{T}_{\mathrm{cs}}$ and filament breakage was quantified by extensive metallographic examinations [11-12].

Strain distribution and filament breakage are interlinked because the breakage will take place in the tensile tail of the strain distribution. Both the compressive and tensile tails of the strain distribution depress $\mathrm{T}_{\mathrm{cs}}$ : the compressive tail via reversible reduction, while the tensile tail via irreversible reduction of $\mathrm{I}_{\mathrm{c}}$. Which tail has the major effect in TF conductors is still debated, see for example the exhaustive review in [13]. Filament 
breakage is often considered responsible of reduction of $\mathrm{T}_{\mathrm{cs}}$ during cycling, but in [14] and [15] it was shown that the broadening of the strain distribution alone (without including breakage) can result in $\mathrm{T}_{\mathrm{cs}}$ reduction during cycling and low $n$ values which were in agreement with the measurements.

The effective strain is often used to characterize CIC. It is a fitting parameter (see for example [14]), used to fit the measurements on conductors with the $\mathrm{I}_{c}(\mathrm{~B}, \mathrm{~T}, \varepsilon)$ measured on the strand. It corresponds to the strain that the strands in the conductors should have to obtain the $\mathrm{I}_{\mathrm{c}}$ and $\mathrm{T}_{\mathrm{cs}}$ effectively measured in the conductor. The $\mathrm{T}_{\mathrm{cs}}$ of a conductor is sensitive to both reversible compressive strain and to filament breakage; hence also the effective strain parameter is affected by both. The effective strain fitting parameter can be used to quantify how far the performance of a conductor is from the one of a free standing strand. In this sense it is well suited to describe the effectiveness of a conductor design.

In 2011 [16] the effective strain of the first qualification samples of ITER TF conductors (20 samples) was estimated to be in the range between $-0.64 \%$ and $-0.89 \%$ after 1000 electromagnetic cycles. In the 2017 release of values [17] regarding the production conductors (over 50 samples) the initial (after one electromagnetic cycle) effective strain was between $-0.60 \%$ and $-0.90 \%$; after 1000 electromagnetic cycles the range is between $-0.62 \%$ and $-0.98 \%$. Over 70 ITER TF conductors have been measured so far in the SULTAN facility at Swiss Plasma Center; the number of sample as a function of effective strain (data from [16] and [17]) is summarized in figure 1: majority of the conductors have effective strain in the $-0.65 \%$ to $0.85 \%$ range. The large variability among samples is due to different sensitivity to fracture and to slightly different strain distribution; in fact all conductors in figure 1 have been manufactured by different suppliers, but according to the same technical specifications. Certainly in future the evaluation of a conductor design should be based on more than a single sample. To summarize: in ITER TF conductors the thermal strain of about $-0.4 \%$ (position of the peak of the strain distribution from $\mathrm{T}_{\mathrm{c}}$ measurements) results in an effective strain between $-0.6 \%$ and $-0.9 \%$. After transverse load cycling the effective strain fitting parameter becomes slightly more negative (less than $-0.05 \%$ ), but this should not prevent the nominal operation of TF magnets during the entire life of ITER because the conductors have sufficient margin. A further warm up / cool down cycle slightly reduce $\mathrm{T}_{\mathrm{cs}}$.

In CS conductors with short twist pitch (no filament breakage) the effective strain lies between $-0.65 \%$ and $0.70 \%$. These values are partially justified by the larger jacket cross section, which apply a larger thermal compression strain and shifts the strain distribution to more negative values.

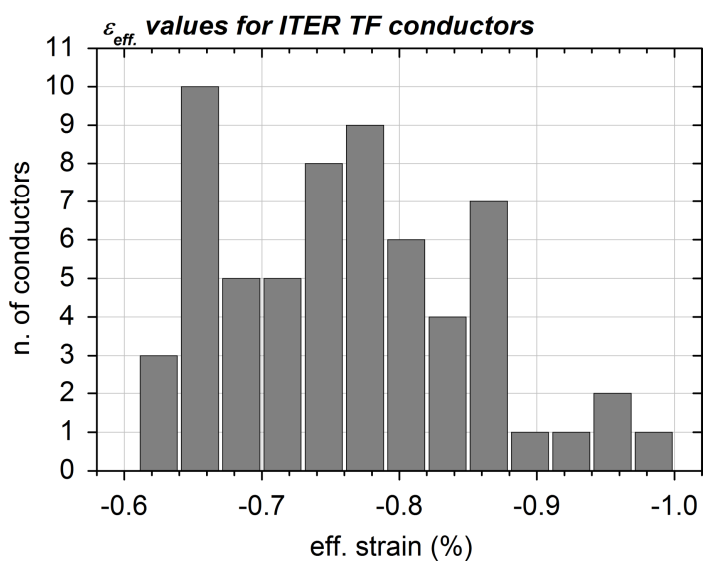

Figure 1. Occurrence (number of conductor) of effective strain on ITER production samples (values from [16-17]) after 1000 electromagnetic cycles 


\subsection{Evaluating the margin of improvement}

The margin of improvement on $\mathrm{T}_{\mathrm{cs}}$ depends on how much the effective strain fitting parameter can be reduced. Indeed there is plenty of room for improvement above the $-0.65 \%$ to $-0.85 \%$ values of ITER conductors. Three reference values of effective strain fitting parameter have been selected to evaluate the margin of improvement on $\mathrm{T}_{\text {cs }}:-0.37 \%,-0.20 \%$ and $0 \%$. The $-0.37 \%$ value is for a hypothetical conductor in which both filament breakage and highly compressed regions are reduced with respect ITER conductors, but the jacket still apply substantial thermal strain. The value of $-0.20 \%$ is for a hypothetical conductor with no filament breakage and no thermal strain from the jacket (thus no strain distribution); in this case the effective strain fitting parameter would be equal to the thermal strain of the wire (around $-0.15 \%$ for Internal Sn wires and around $-0.25 \%$ for Bronze Route wires). Finally the highest $\mathrm{T}_{\mathrm{cs}}$ would be obtained in a hypothetical CIC with effective strain fitting parameter of $0 \%$, when not only filament breakage is absent but even the thermal strain from the strand matrix vanishes; this is clearly not implementable in a real coil because the hoop strain may induce fracture in the wire. Also values between $-0.2 \%$ and $0 \%$ are hardly implementable in large fusion magnets, but are achieved in commercial high field solenoids (like NMR magnets) where the hoop strain compensate the matrix compression on the $\mathrm{Nb}_{3} \mathrm{Sn}$ filaments. In high field accelerator magnets (wound with Rutherford cables) there is no need to introduce an effective strain.

Four TF samples have been selected for these estimations: TFEU4 and TFEU13, which had one of the highest $\mathrm{T}_{\text {cs }}$ and lowest effective strain; two RF conductors (RF6 right and left samples), because the RF conductor are the only ones that do not show cycling reduction of $\mathrm{T}_{\mathrm{cs}}$, probably because of the Cr plating roughness, as suggested in [14]; EUTF10, which had one of the most negative effective strain. These few conductors are representative of the whole production in the sense that their effective strain values cover the whole range reported in figure 1. The measured current sharing temperatures as a function of electromagnetic cycling are reported in figure $2 \mathrm{abcd}$. In the same figure the expected $\mathrm{T}_{\mathrm{cs}}$ for hypothetical conductor with effective strain fitting parameters of $-0.37 \%,-0.2 \%$, and $0 \%$, have been calculated following the procedure described in [18] and plotted: all these values lie well above $(1 \mathrm{~K}$ to $3 \mathrm{~K})$ the measured values. Similar values are obtained also for CS conductors (short twist pitch).

Since early 2000' the transverse load on the cable has been considered the main responsible for the reduction of $\mathrm{T}_{\mathrm{cs}}$ during cycling; this hypothesis lead to the development of methods to better support the strand in the cable against transverse load, for example by reducing the void fraction, adjusting the twist pitches of the stages (for example, the cycling reduction has been suppressed in CS conductor by shortening the twist pitch of all but the last cabling stages), changing the aspect ratio of the cable (in a rectangular cable the transverse pressure is lower than in a round one).It should be pointed out that both simulations [9] and measurements [10] show that the large strain distribution, and thus large effective strain and depressed $\mathrm{T}_{\mathrm{cs}}$, is present already after the first cooldown, before any transverse load is applied.

The optimization of the void fraction and of the twist pitches have a beneficial, but modest, impact on $\mathrm{T}_{\mathrm{cs}}$ and were incorporated in the new ITER design [19]. In fact (see fig 2 in [13]) in CS conductors with standard twist pitch (prone to extensive filament breakage), the $T_{c s}$ reduction after 1000 cycles is $<0.5 \mathrm{~K}$ and the initial values of $\mathrm{T}_{\mathrm{cs}}$ is comparable to the one measured in the CS conductor with short twist pitches (no filament breakage), whose $\mathrm{T}_{\mathrm{cs}}$ is constant with cycling.

In TF conductors the degradation during cycling is also small ( $<0.5 \mathrm{~K}$ in fig. $2 \mathrm{abcd})$. The RF conductors, which have no cycling degradation despite filament breakages, still have a very depressed $\mathrm{T}_{\mathrm{cs}}$ (over $2 \mathrm{~K}$ ) compared to the values for uniform strain of $-0.37 \%,-0.2 \%$ and $0 \%$ (see fig. $2 \mathrm{c}$ ).

The degradation of $\mathrm{T}_{\mathrm{cs}}$ observed in TF conductors has been taken into account in the design of ITER and it will not prevent the normal operation of ITER. If one is open to reconsider the whole design of the conductor, aiming for example to reduce the effective strain fitting parameter to $-0.3 \% \sim-0.4 \%$, the gain could be very 
large, few Kelvin, as shown in fig. 2abcd and discussed in [20]. The margin of improvement is basically two to three times larger than the reduction observed after 1000 electromagnetic cycles in TF conductors.
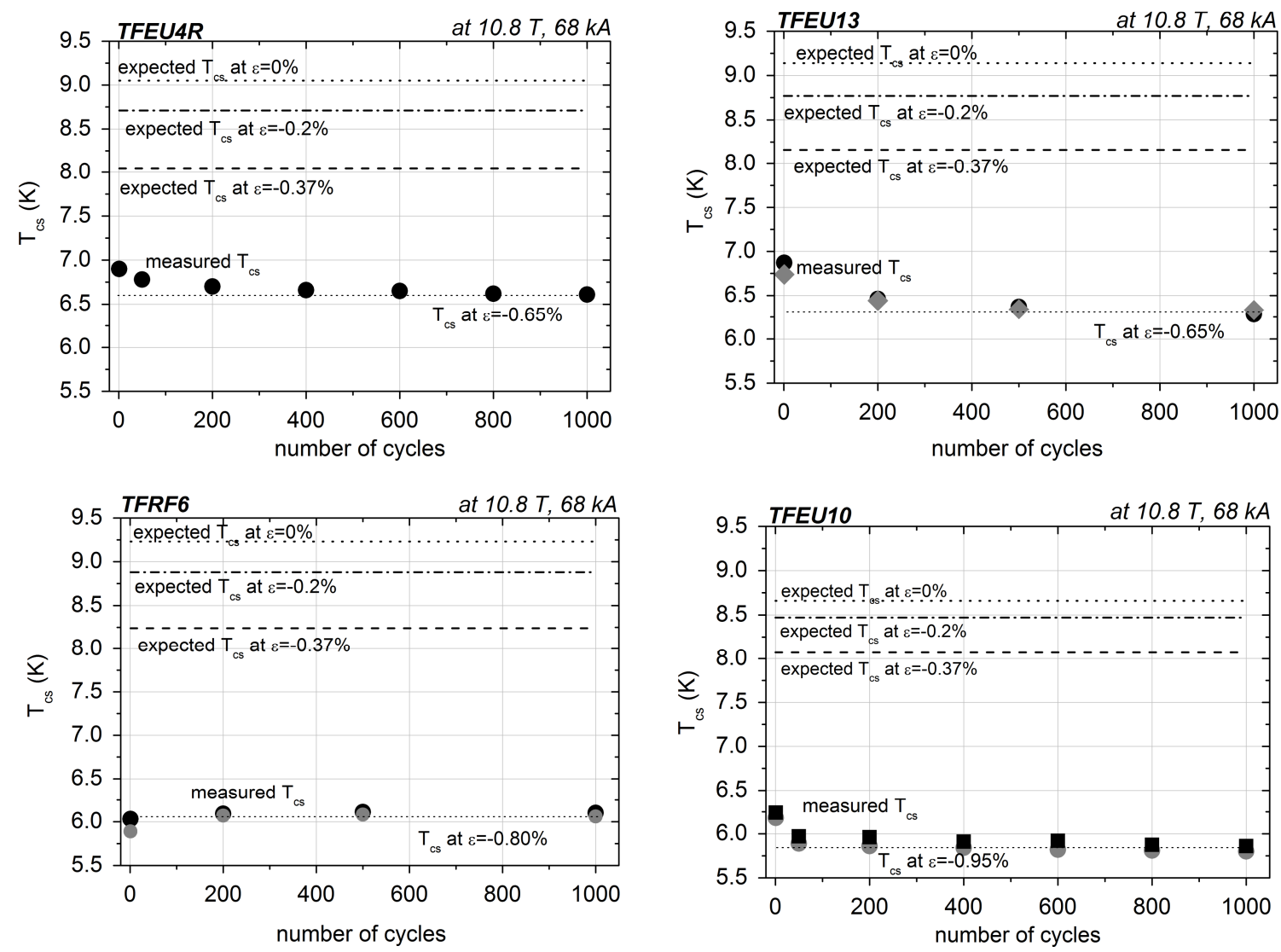

Figure 2abcd. Measured $\mathrm{T}_{\mathrm{cs}}$ for ITER TF conductors: TFEU13 right and left legs, TFEU4R right leg, TFRF6 right and left legs, TFEU10 right and left legs. The acceptance criterion is $5.8 \mathrm{~K}$. The effective strain (values from [12] and [13]) are also reported. The dashed lines are for hypothetical conductors made with the same strands but working at effective strain of $0.37 \%,-0.2 \%$ and $0 \%$.

\section{Review of DEMO prototype conductors}

In Europe the EUROfusion Consortium is carrying out the conceptual R\&D for the DEMO reactor. In the activities regarding the magnet system development [21], three alternative conductors for the TF magnet are being investigated by CEA, ENEA and SPC.

CEA has prepared the design of a square conductor [22], assuming a design effective strain of $-0.66 \%$, which has been observed in some ITER TF conductors; the winding pack will consist of W\&R double pancakes, as in ITER TF magnet. The fabrication of a prototype conductor is in preparation.

The ENEA conductor (ENEA-WR1) is a W\&R conductor based on the alternative TF conductor presented in 2010 [23]: in contrast with the TF ITER conductor, it has a rectangular cross section (see [24] for details), which reduce the transverse pressure on the cable, and a smaller void fraction (higher compaction); the winding pack will consist of double layers and thus will be wound with a graded conductor. The high grade conductor was manufactured industrially. The test showed no reduction of the $\mathrm{T}_{\mathrm{cs}}$ during cycling and the effective strain was estimated to be between $-0.5 \%$ and $-0.55 \%$ [24].

The SPC conductors (SPC-RW1 and 2) are based on the R\&W conductor developed in 2007 [25], whose effective strain was estimated at $-0.28 \%$. The winding pack is layer wound, allowing the use of a graded 
conductor. The conductors are composed of a flat cable with low void fraction which is reacted before jacketing; therefore the thermal compression exerted by the steel jacket on the $\mathrm{Nb}_{3} \mathrm{Sn}$ cable acts only from room temperature to $4.2 \mathrm{~K}$, instead of starting close to the reaction temperature. The high field conductors were manufactured industrially and the effective strain fitting parameter was estimated between $-0.28 \%$ and $0.32 \%$ in SPC-RW1 [26] (in this sample the jacket was only $3 \mathrm{~mm}$ thick). Recently [27] a second prototype, SPC-RW2, designed for different operating conditions, has been manufactured and tested; the effective strain fitting parameter shifted from an initial value of $-0.35 \%$ to $-0.40 \%$ after 1000 cycles. The value is larger than in SPC-RW1 probably because of the much thicker steel jacket.

All these conductors were manufactured with an Internal Sn strand produced by Western Superconducting Technologies (WST); the strand is very similar to the one used for the Chinese ITER TF conductors, the main difference is the larger diameter. The $n$ values are in the range $7 \sim 10$ for ENEA-RW1 and up to 18 for SPCRW2; for comparison in CS conductor the $n$ values were up to 12 while in TF conductors values as low as 5 were measured. In contrast with ITER TF conductors, the prototype DEMO conductors do not show reduction of $\mathrm{T}_{\mathrm{cs}}$ after warm-up cool-down cycle.

A metallographic examination of the conductors has not yet been carried out, therefore the amount of filament fractures is unknown. The measurement of strain distribution in the ENEA and SPC conductors is planned in 2018, but simulations (see fig 5.11 in [9]) showed that the strain distribution gets narrower and shift to less negative values when the applied axial compression is lower. Therefore, at least for the R\&W conductors, the strain distribution is expected to be smaller than in ITER conductors.

Despite the lack of information on fractures and strain distribution, in the DEMO prototypes the effective strain fitting parameter is less negative than in ITER TF and CS (short twist pitch) conductors, indicating that the strand can operate at higher current. In table I the operating conditions, non-Cu cross sections and operating current densities are reported for ITER and DEMO conductors. The effect of less negative effective strain fitting parameter is not clearly visible among different conductors, because of the different operating conditions. Nevertheless it appears that the SPC-RW2 conductor, which has lower effective strain fitting parameter than ITER TF conductors, can carry a similar current at a larger temperature margin with a smaller non-Cu cross-section. This comparison will be further developed in section 4 .

EUROfusion has asked to CEA, ENEA and SPC to develop conceptual designs not only of the conductor but also of the whole winding pack; nevertheless it should be stressed that the conductor design is in principle independent from the design of the winding pack: for example a $\mathrm{R} \& \mathrm{~W}$ conductor like the one proposed by SPC could be used in a non-graded, double pancake winding pack; conversely a W\&R conductor like the CEA one could be used for a graded and layer-wound winding pack. Of course the detailed design of the conductor should be optimized for the specific winding pack.

It is clear that the maturity of the above mentioned DEMO prototype conductors is not yet at the level that has been reached at the end of the ITER R\&D phase. Before advanced conductors could be successfully used in the construction of a large magnet system more development is needed. Certainly more conductors should be manufactured and tested with different strands (Bronze / Internal Sn Routes, various manufacturers) to verify the performance, in fact in ITER TF conductors the range of effective strain values kept broadening as more conductors were manufactured and tested. Finally the manufacture and test of a model coil is required before the validation of a new conductor design and winding pack, as it was done for ITER with the TF and CS Model Coils and later with the TF and CS Inserts. 
Relation between non-Cu cross sections and $\varepsilon_{e f f}$ for ITER and prototype DEMO conductors

\begin{tabular}{|c|c|c|c|c|c|}
\hline & $\begin{array}{l}\text { Operating } \\
\text { current and } \\
\quad \text { field }\end{array}$ & $\begin{array}{l}\text { Design temp. } \\
\quad \text { margin }\end{array}$ & $\begin{array}{l}\text { Non- } \mathrm{Cu} \text { cross } \\
\text { section }\end{array}$ & $\begin{array}{c}\text { Operating } \\
\text { current } \\
\text { density }\end{array}$ & $\varepsilon_{\text {eff }}$ fitting par. \\
\hline ITER TF & $68 \mathrm{kA}, 11.8 \mathrm{~T}$ & $0.7 \mathrm{~K}$ & $226 \mathrm{~mm}^{2}$ & $300 \mathrm{~A} / \mathrm{mm}^{2}$ & $-0.62 \% \sim-0.92 \%$ \\
\hline ITER CS & $45 \mathrm{kA}, 13 \mathrm{~T}$ & $0.7 \mathrm{~K}$ & $145 \mathrm{~mm}^{2}$ & $310 \mathrm{~A} / \mathrm{mm}^{2}$ & $-0.65 \% \sim-0.71 \%$ \\
\hline DEMO TF, SPC-RW1 & $82 \mathrm{kA}, 13.5 \mathrm{~T}$ & $1.5 \mathrm{~K}$ & $270 \mathrm{~mm}^{2}$ & $300 \mathrm{~A} / \mathrm{mm}^{2}$ & $-0.28 \% \sim-0.30 \%$ \\
\hline DEMO TF, ENEA-WR1 & $82 \mathrm{kA}, 13.5 \mathrm{~T}$ & $1.5 \mathrm{~K}$ & $424 \mathrm{~mm}^{2}$ & $190 \mathrm{~A} / \mathrm{mm}^{2}$ & $-0.50 \% \sim-0.55 \%$ \\
\hline DEMO TF, CEA & $87 \mathrm{kA}, 13.5 \mathrm{~T}$ & $1.5 \mathrm{~K}$ & $522 \mathrm{~mm}^{2}$ & $170 \mathrm{~A} / \mathrm{mm}^{2}$ & $-0.66 \%^{\mathrm{a}}$ \\
\hline DEMO TF ${ }^{\mathrm{b}}$, SPC-RW2 & $63 \mathrm{kA}, 12.2 \mathrm{~T}$ & $1.5 \mathrm{~K}$ & $132 \mathrm{~mm}^{2}$ & $475 \mathrm{~A} / \mathrm{mm}^{2}$ & $-0.35 \% \sim-0.40 \%$ \\
\hline
\end{tabular}

Table I. Operating conditions and non-Cu cross sections for ITER TF, CS conductors and for prototype DEMO TF conductors. In all conductors the $\mathrm{Cu}$ :nonCu ratio of the strands is $1 .{ }^{\mathrm{a}}$ Design values, conductor not yet tested. ${ }^{\mathrm{b}}$ EUROfusion 2015 baseline.

\section{Discussion}

In section 2 it was shown that conductors with less negative effective strain than ITER TF conductors would deliver a much higher current sharing temperature, resulting in a very large temperature margin; such high temperature margins, well above the design values, have limited practical advantage. It would actually be more valuable, in view of commercial application of fusion, to bring back the temperature margin to the design value by reducing the number of $\mathrm{Nb}_{3} \mathrm{Sn}$ strands, i.e. reducing the amount and thus the cost of $\mathrm{Nb}_{3} \mathrm{Sn}$. Therefore, it is meaningful to evaluate the margin of improvement for a conductor not only in term of current sharing temperature but rather in term of critical current of $\mathrm{Nb}_{3} \mathrm{Sn}$ strands. A similar analysis was carried out for ITER TF conductors (see figure $10 \mathrm{in} \mathrm{[17])} \mathrm{and} \mathrm{it} \mathrm{was} \mathrm{found} \mathrm{that,} \mathrm{after} \mathrm{setting} \mathrm{the} \mathrm{effective} \mathrm{strain} \mathrm{at}-0.5 \%$, the corresponding critical current was found to be about only $50 \%$ of the values expected for uniform strain.

\subsection{Low cost DEMO}

A more direct way of presenting the margin of improvement is to consider the strain dependence of the critical current. In figure 3 the normalized critical current as a function of strain has been plotted for five strands (see [28]) used in the production of the ITER conductors. The parameters in [28] were determined from measurements in a wide range of strain $(-0.9 \%$ to $+0.4 \%$ applied), temperature (4.2 12 K) and magnetic field (4 14 T) and are thus reliable fit of the experimental values. The curves have been calculated using ITER fitting parameters and scaling law [29] and the magnetic field and temperature are the ones relevant for large tokamaks, i.e. $12 \mathrm{~T}$ and $6 \mathrm{~K}(4.5 \mathrm{~K}$ at the helium inlet plus $1.5 \mathrm{~K}$ of temperature margin). In figure 3 the strain range of the ITER TF and CS conductors has been reported together with the ranges of the ENEA-WR1 and SPC-RW1/SPC-RW2 prototype conductors. In ITER TF conductors the strand critical current is only $10 \%$ to $50 \%$ of the maximum achievable value (around 35\% for CS conductors), while in the ENEA-WR1 the strands have retained from $47 \%$ to $62 \%$ of the maximum critical current (almost 2 times higher than the average value for ITER TF conductors) and in the SPC-RW1 and SPC-RW2 the strands could retain from $65 \%$ to $85 \%$ of the maximum achievable critical current (about 2 to 2.5 times higher than in ITER TF conductors and about 2 times higher than in CS conductors).

Figure 3 provides a clear proof that a conductor design which would use in a more efficient way (i.e. at less negative effective strain) the $\mathrm{Nb}_{3} \mathrm{Sn}$ could need up to 4 times less strands than the ITER conductors in the high 
field region. This is also supported by the values of table 1: conductors with low effective strain (even if they guarantee higher temperature margins) tend to operate at higher current density and therefore need less $\mathrm{Nb}_{3} \mathrm{Sn}$ strand (smaller non-Cu cross section).

Independently of the conductor design, a further saving in superconducting strands can be obtained by using a graded conductor, i.e. reducing the amount of strands in the winding pack section where the magnetic field is lower. Grading is generally considered more suitable for layer wound winding, but it is in principle applicable to double pancakes winding (for example the TRIAM1M tokamak, see [30]) if a convenient joining technique is developed. In TF magnet, like that of EU-DEMO, the use of a graded winding pack could save up to $50 \%$ of the $\mathrm{Nb}_{3} \mathrm{Sn}$ (see for example the winding pack proposed in [26]) compared to a non-graded magnet. One more advantage of grading is that the strands with the lowest performance, and eventually lowest cost, could be used in the low field regions, without affecting the overall performance of the TF magnet system. In a TF layer wound coils the first layer will collect more nuclear heat load than in double pancake and will require a slightly larger mass flow rate of $\mathrm{He}$, as incorporated in the design (see [27])

The industrial feasibility of large coil (layer wound and double pancake, W\&R and R\&W conductors) is presently being investigated in the EUROfusion DEMO activities, for example a specific work package is aiming to develop joints that can be included into a layer.

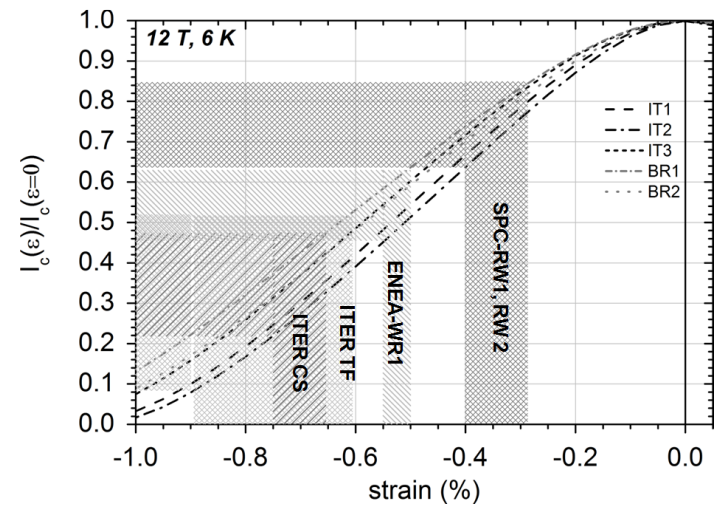

Figure 3. Reduced critical current as a function of the effective strain calculated for five strands, IT1, IT2, IT3, BR1, and BR2 (from [24]). The ranges of effective strain for different conductors are also indicated.

The TF magnet of ITER required about $380 \mathrm{t}$ of strand. For EU-DEMO TF magnet, in case of a non-graded winding pack with ITER type conductors (about $-0.70 \%$ effective strain fitting parameter, for example as in short twist pitch CS conductor), about $850 \mathrm{t}$ will be needed. The effect of grading and improved conductor can be evaluated as follow: in case of grading, and using the same ITER type conductor, the amount could be reduced by a factor two to about $430 \mathrm{t}$ to $560 \mathrm{t}$. In case of a non-graded winding pack (for example double pancake winding) if a more efficient conductor (about $-0.4 \%$ effective strain instead of $-0.75 \%$ ) is employed the amount could be reduced by a factor 2 to 2.5 resulting in about 330 t to $420 \mathrm{t}$ of $\mathrm{Nb}_{3} \mathrm{Sn}$. In the most favourable situation, the combination of a conductor with about $-0.4 \%$ effective strain and of a graded winding, the amount of $\mathrm{Nb}_{3} \mathrm{Sn}$ would be reduced by a factor 4 to 5 (a factor 2 to 2.5 from improved conductor and a factor 2 from grading) resulting is about $220 \mathrm{t}$ to $280 \mathrm{t}$; these values are summarised in fig. 4 . The validity of this rough estimate is confirmed by the values reported in a detailed winding pack analysis in [26]. Therefore the TF magnet of DEMO could be built with less $\mathrm{Nb}_{3} \mathrm{Sn}$ than the one of ITER, despite the much larger size of the DEMO TF magnet.

In case of EU-DEMO, a saving of just $50 \%$ on the $\mathrm{Nb}_{3} \mathrm{Sn}$ strand would correspond to about 100 to 200 million of euros (assuming a strand price of 600 to 1000 euro $/ \mathrm{kg}$ ); in the most favourable case (corresponding to a factor four reduction in $\mathrm{Nb}_{3} \mathrm{Sn}$ ) the saving would exceed 500 million euro. 


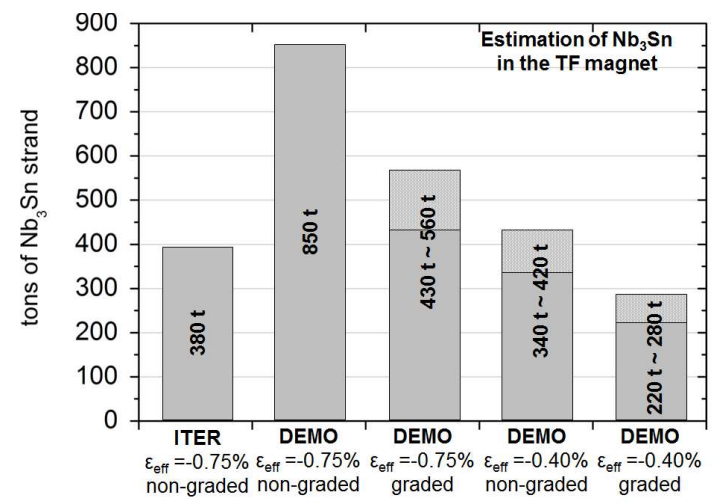

Figure 4. Estimation of amount of $\mathrm{Nb}_{3} \mathrm{Sn}$ strand required for DEMO TF magnet system depending on the type of winding pack (graded/non-graded) and the effective strain fitt. parameter of the conductor.

\subsection{High field and low cost DEMO}

A conductor with a much lower effective strain would not only bring a clear economic benefit but it would also enable operation at magnetic fields higher than the ones of ITER type conductors. In fact the irreversibility field of $\mathrm{Nb}_{3} \mathrm{Sn}$ depends on the strain; it follows that the irreversibility field and thus the $\mathrm{I}_{\mathrm{c}}$ of a $\mathrm{Nb}_{3} \mathrm{Sn}$ strand operating at a very high compressive strain is strongly diminished compared to the operation at less negative strain. This effect is shown in figure 5, where the critical current versus strain at $6 \mathrm{~K}$ is plotted for the strands from [28]: the same critical current obtained around $-0.75 \%$ at $12 \mathrm{~T}$ can be obtained at higher field, for example at $15 \mathrm{~T}$, at a lower strain, around $-0.40 \%$.

The use of such conductor makes it possible to build a DEMO tokamak generating a higher peak field, and thus higher field on the plasma. This has a striking consequence on the design of the tokamak because at constant fusion power the volume of a tokamak scales roughly like $\mathrm{B}^{3}$ [31]; therefore, for the same fusion power in burning conditions, an increase of just $25 \%$ (from about $12 \mathrm{~T}$ to about $15 \mathrm{~T}$ ) in peak field, and thus in the plasma field, may allow for up to $60 \%$ to $65 \%$ reduction of the volume of the tokamak. In a higher field, more compact tokamak not only the amount of $\mathrm{Nb}_{3} \mathrm{Sn}$ would be reduced, but also the steel structures, the vacuum vessel, the blanket (all very expensive components) would be substantially reduced with respect to a $12 \mathrm{~T} \sim 13 \mathrm{~T}$ tokamak. Therefore the increase in operating field will bring a larger cost reduction than simply using an improved conductor in a $12 \mathrm{~T} \sim 13 \mathrm{~T}$, large tokamak.

The advantages and disadvantages of compact, high field tokamak are still being evaluated. In favour, it has been pointed out (see [28]) that the increase in B provides even more margin from disrupting operation limits. An argument against compact/high field tokamak is that the extrapolated heat and neutron fluxes at the divertor would be too high. Nevertheless the validity of the extrapolation for high power tokamak is still debated and a definitive answer may not come before the D-T operation of ITER (around 2030-2035). Here it should be stressed that, if the exhaust management at the divertor is less critical than expected, a $15 \mathrm{~T}$ tokamak, substantially more compact (and thus cheaper) than a $12 \mathrm{~T}-13 \mathrm{~T}$ tokamak like EU-DEMO could be built with "traditional" $\mathrm{Nb}_{3} \mathrm{Sn}$, without the need to use HTS wires or tapes, as proposed for much more ambitious projects like ARC from MIT [31] or the Spherical Tokamak from Tokamak Solution [32]. In contrast with high performance $\mathrm{Nb}_{3} \mathrm{Sn}$ conductors, novel HTS conductors may be not available in sufficient quantity/quality and would require a much larger $R \& D$ effort before being ready to be used in a demonstration fusion plant. 


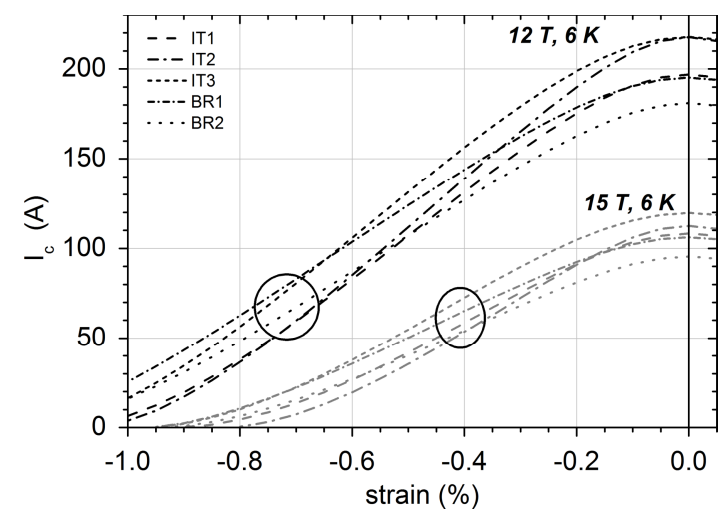

Figure 5. Critical currents versus strain at $6 \mathrm{~K}, 12 \mathrm{~T}$ and $15 \mathrm{~T}$ for strands BR1, BR2, IT1, IT2, IT3 from [24]. The circles indicate that similar $\mathrm{I}_{\mathrm{c}}$ can be obtained at $12 \mathrm{~T},-0.75 \%$ effective strain fitt. parameter or at $15 \mathrm{~T},-0.4 \%$ effective strain fitt. parameter.

\section{Paths to CIC improvements}

The ENEA and SPC conductors are only examples of alternative conductors with improved performance. Two old designs that could be further developed and tested are: monolithic conductors, in which the strands are soldered together with a soft solder and conductors whose jacket has a thermal contraction coefficient very close to the one of the $\mathrm{Nb}_{3} \mathrm{Sn}$ strands.

Even the rope cable ITER design may be still further improved. In fact, among the prototype ITER conductors, the TFPRO2 with long twist pitches (see fig. 3 in [33]) stands out not only for the constant $T_{c s}$ but also for the high initial $\mathrm{T}_{\mathrm{cs}}$ value, resulting in a remarkable effective strain fitting parameter of about $-0.4 \%$ (see [34]), a value comparable to the ones observed in R\&W conductors (SPC-RW2). Therefor this option should be reconsidered despite the unsuccessful results observed in CSIO with long twist pitches. Finite element analysis could help to clarify the influence of twist pitches and void fraction on strain distributions and to narrow down possible successful designs, without following a "trial and error" strategy which requires expensive manufacturing and test of conductors.

One more possibility to further cut cost is to move some of the copper from the strand to segregated wires; ITER already moved from a Cu:non-Cu of 1.5 to 1 , which is also used in the DEMO conductors. It should be verified if the $\mathrm{Cu}$ :non- $\mathrm{Cu}$ ratio could be further reduced without compromising the stability.

Tools like finite element analysis of cables to evaluate strain distribution (for example Multifil [9] or simpler like FEMCAM [15]), inductive $T_{c}$ measurements and metallographic examinations were introduced only at the very end of the ITER conductor R\&D program, when the production of strand and conductors was already started, leaving very limited freedom to change the conductor design. These tools could play a very important role if used since the beginning of a new conductor development. DEMO construction in Europe is planned to start not before 2035 and there is certainly plenty of time to design, fabricate and test several advanced conductors.

In new conductors several requirements must be fulfilled, like low AC losses, stability, thermo-hydraulic characteristics, industrial feasibility and joints. Some of these are already being investigated in the DEMO EUROfusion program. The cost of such R\&D program could be of the order of few millions of euros; this investment in $R \& D$ is however a small price to pay considering the hundreds of millions that can be saved in the procurement of the $\mathrm{Nb}_{3} \mathrm{Sn}$ strands. It should be also stressed that the largest step in technical and engineering point of view was to go from $\mathrm{NbTi}$ to $\mathrm{Nb}_{3} \mathrm{Sn}$, and this step has already been accomplished by ITER; the next step, from ITER conductor to a more effective alternative, would very likely require a smaller investment and R\&D effort. 


\section{Conclusions}

ITER $\mathrm{Nb}_{3} \mathrm{Sn}$ conductors have been successfully manufactured across three continents setting new records in terms of amount of $\mathrm{Nb}_{3} \mathrm{Sn}$ produced. The conductors will guarantee the correct operation of ITER magnet system during the entire life of the machine.

Nevertheless ITER conductors still have a large margin of improvement because of the combined effect of compressive tail of strain distribution and filament breakage (in TF conductors only). This effect can be quantified using the effective strain fitting parameter. The strand critical current in ITER conductors is reduced to values between $15 \%$ and $50 \%$ of the maximum value at $12 \mathrm{~T}, 6 \mathrm{~K}$.

Experimental conductors built in 2008-10 (for example TFPRO2) and recent prototype conductors designed and manufactured for EU-DEMO have proven that it is possible to rise the performance (i.e. reducing the effective strain fitting parameter); an improved conductor would need less than half of the $\mathrm{Nb}_{3} \mathrm{Sn}$ strands needed for ITER conductor, simply because the strands could operate at a current density twice higher than the one of ITER conductors. Further reduction in the amount of $\mathrm{Nb}_{3} \mathrm{Sn}$ can be achieved by using graded conductors; in the best case (less negative effective strain and grading) the TF magnet system of EU-DEMO would require less $\mathrm{Nb}_{3} \mathrm{Sn}$ than the one of ITER.

In addition, advanced conductors open up the possibility to build compact, higher field tokamak achieving even larger cost reduction, because peak fields up to about $15 \mathrm{~T}$ could be achieved.

The development of alternative conductors and winding packs would cost few millions euros; this investment is clearly very profitable, because it would allow saving hundreds of tons of $\mathrm{Nb}_{3} \mathrm{Sn}$, whose value is hundreds of millions of euro. For the economic viability of fusion power it is highly desirable that advanced conductors are developed for future large tokamaks (like EU-DEMO, CFTER, and K-DEMO), instead of relying on the proven and reliable but less cost effective ITER type conductors.

\section{Acknowledgment}

The authors wish to thank H. Reimerdes (Swiss Plasma Center, EPFL) for the fruitful discussion on tokamaks and divertors. The authors wish to thank T. Boutboul (F4E) and V. Tronza (ITER) for providing the permission to use the data of the conductors manufactured by the European and Russian Domestic Agencies.

\section{References}

[1] Bruzzone P 200630 Years of Conductors for Fusion: A Summary and Perspectives IEEE Trans. on App. Supercond. 16 839-844

[2] Fusion Electricity - A roadmap to the realisation of fusion energy 2012 European Fusion Development Agreement, EFDA () francesco romanelli (EFDA Leader) ISBN 978-3-00-040720-8 https://www.euro-fusion.org/wpcms/wpcontent/uploads/2013/01/JG12.356-web.pdf

[3] Aymar R et al 2001 Summary of the ITER final design report IAEA/ITER EDA/DS/22, Printed by the IAEA in Vienna, http://www-pub.iaea.org/MTCD/Publications/PDF/ITER-EDA-DS-22.pdf

[4] Mitchell N and Bottura L 1991 Superconductors for the NET Coils Fusion Eng. and Design 15 85-100

[5] Ciazynski D et al 2000 Test Results and Analysis of Two European Full-Size Conductor Samples for ITER IEEE Trans. on App. Supercond. 10 1058-1061

[6] Mitchell N 2003 Summary, assessment and implications of the ITER model coil test results Fusion Eng. Des. 66-68 971-993

[7] Ciazynski D 2007 Review of $\mathrm{Nb}_{3}$ Sn conductors for ITER” Fusion Eng. and Design $82488-497$

[8] Zanino R, Mitchell N and Savoldi Richard L. 2003 Analysis and Interpretation of the Full Set (2000-2002) of Tcs Tests in Conductor 1A of the ITER Central Solenoid Model Coil Cryogenics 43 179-197

[9] Bajas H 2011 Numerical simulation of the mechanical behavior of the ITER cable-in-conduit conductors Ecole Centrale Paris, PhD. thesis https://hal.inria.fr/tel-00697000/document

[10] Calzolaio C and Bruzzone P 2014 Analysis of the CICC Performance Through the Measurement of the Thermal Strain Distribution of the $\mathrm{Nb}_{3} \mathrm{Sn}$ Filaments in the Cable Cross Section IEEE Trans. on Appl. Supercond. 244802204 
[11] Sanabria C et al 2015 Metallographic autopsies of full-scale ITER prototype cable-in-conduit conductors after full cyclic testing in SULTAN: II. Significant reduction of strand movement and strand damage in short twist pitch CICCs Supercond. Sci. Technol. 28125003

[12] Sanabria C et al. 2016 Metallographic autopsies of full-scale ITER prototype cable-in-conduit conductors after full cyclic testing in SULTAN: III. The importance of strand surface roughness in long twist pitch conductors Supercond. Sci. Technol. 29074002

[13] Mitchell N et al 2013 Reversible and irreversible mechanical effects in real cable-in-conduit conductors Supercond. Sci. Technol. 26114004

[14] Breschi M, Ribani P L, Bajas H and Devred A 2012 Modeling of the electro-mechanical behavior of ITER Nb3 $\mathrm{Sn}_{3}$ cable in conduit conductors Supercond. Sci. Technol. 25054005

[15] Zhai Y 2010 Electro-mechanical modeling of $\mathrm{Nb}_{3} \mathrm{Sn}$ CICC performance degradation due to strand bending and inter-filament current transfer Cryogenics 50 149-157

[16] Breschi M, Bessette D and Devred A 2011 Evaluation of Effective Strain and n-Value of ITER TF Conductor Samples IEEE Trans. on Appl. Supercond. 211969

[17] Breschi M et al. 2017 Performance analysis of the toroidal field ITER production conductors Supercond. Sci. Technol. 30055007

[18] Wesche R et al. 2009 Results of conductor testing in SULTAN: A review Proceedings of WAMSDO-Accelerator Magnet Superconductors, Design and Optimization 2009-001 68-77

[19] Devred A et al. 2014 Challenges and status of ITER conductor production Supercond. Sci. Technol. 27044001

[20] Muzzi L et al 2015 Cable-in-conduit conductors: lessons from the recent past for future developments with low and high temperature superconductors Supercond. Sci. Technol. 28053001

[21] Zani L et al. 2016 Overview of progress on the EU DEMO reactor magnet system design IEEE Trans. on Appl. Supercond. 264204505

[22] Torre A, Ciazynski D, Hertout P, and Zani L 2016 Tools Used at CEA for Designing the DEMO Toroidal Field Coils Winding Pack IEEE Trans. Appl. Supercond. 264902005

[23] Della Corte A et al. 2010 Successful performances of the EU-AltTF sample, a large size $\mathrm{Nb}_{3} \mathrm{Sn}$ cable- in-conduit conductor with rectangular geometry Supercond. Sci. Technol. 23045028

[24] Muzzi L et al. 2017 Design, Manufacture, and Test of an 80 kA-Class Nb3Sn Cable-In-Conduit Conductor With Rectangular Geometry and Distributed Pressure Relief Channels IEEE Trans. on Appl. Supercond. 274800206

[25] Bruzzone P, Bagnasco M, Cau F, Stepanov B and Wesche R 2008 Development of a react and wind conductor for the ITER toroidal field coils IEEE Trans. Appl. Supercond. 18 467-470

[26] Sedlak K, Bruzzone P, Sarasola X and Stepanov B 2016 Design and R\&D for the toroidal field coils of DEMO by react and wind method IEEE Trans. Appl. Supercond. 274800105

[27] Sedlak K et al. A Prototype Conductor by React\&Wind Method for the EUROfusion DEMO TF Coils Presented at MT25, to be published in 2018 IEEE Trans. Appl. Supercond. 28

[28] Nijhuis A et al 2013 The effect of axial and transverse loading on the transport properties of ITER $\mathrm{Nb}_{3} \mathrm{Sn}$ strands Supercond. Sci. Technol. 26084004

[29] Bottura L and Bordini R $2009 \mathrm{~J}_{\mathrm{c}}(\mathrm{B}, \mathrm{T}, \varepsilon)$ parameterization for the ITER Production IEEE Trans. Appl. Supercond. 19 1521

[30] Sakamoto M et al. 1999 Effect of plasma disruption on superconducting magnet system in TRIAM-1M Fusion Engineering and Design 47 1-7

[31] Whyte D G et al. 2016 Smaller \& Sooner: Exploiting High Magnetic Fields from New Superconductors for a More Attractive Fusion Energy Development Path J Fusion Energ 35 41-53

[32] Gryaznevich M P et al. 2015 Advancing Fusion by Innovations: Smaller, Quicker, Cheaper Journal of Physics: Conference Series, 591, 012005

[33] Ciazynski D et al 2008 Influence of Cable Layout on the Performance of ITER-type Nb3Sn Conductors J. Phys.: Conf. Ser. 97012027

[34] van Lanen E P A and Nijhuis A 2011 Numerical analysis of the DC performance of ITERTF samples with different cabling pattern based onresistance measurements on terminations Supercond. Sci. Technol. 24085010 